\title{
Validez del Maslach Burnout Inventory General Survey en conductores informales del transporte urbano en México
}

Validez del Maslach Burnout Inventory General Survey en conductores informales del transporte urbano en México

Francisco Javier, Ortega Sánchez Universidad Autónoma Del Estado de Morelos,

México

ortegasfj@outlook.com

https://orcid.org/0000-0001-6595-5148

Arturo, Juárez García Universidad Autónoma Del Estado de Morelos,

México

arturojuarez@hotmail.com

https://orcid.org/0000-0003-3264-679X

César, Merino Soto Universidad de San Martín de Porres, Perú sikayax@yahoo.com.ar

https://orcid.org/0000-0002-1407-83063

Para referenciar este artículo:

Ortega, F. J. y Juarez, A. y Merino-Soto, C. (2020). Validez del Maslach Burnout Inventory General Survey en conductores informales del transporte urbano en México. Revista ConCiencia EPG, 5(2), 70-

https://doi.org/10.32654/CONCIENCIAEP G.5-2.5

\section{Resumen}

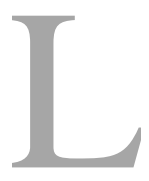

os conductores del transporte urbano de pasajeros que se encuentran dentro de la economía informal en México parecen especialmente vulnerables a diversas consecuencias psicosociales entre ellas el síndrome de Burnout, sin embargo, la evidencia psicométrica de las medidas utilizadas para su evaluación es prácticamente inexistente en este sector. Por ello, en el presente estudio se analiza la consistencia interna y la validez de puntajes del MBI-GS (Maslach Burnout Inventory, General Survey), en una muestra de conductores del transporte urbano de pasajeros en México. La consistencia interna fue adecuada para las tres dimensiones (>.70). El Análisis Factorial Confirmatorio reproduce satisfactoriamente la estructura trifactorial del MBI-GS (CFI = .989, RMSEA $=.045$, SRMR $=.075$ ) y se observan correlaciones de bajas a moderadas para la validez de criterio con trastornos (síntomas) psiquiátricos no psicóticos (GHQ-28). Se discuten las implicaciones de los resultados.

Palabras Clave: Burnout; validez; análisis factorial confirmatorio; consistencia interna. 


\section{Abtract}

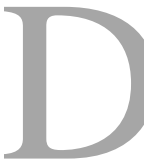

rivers of urban passenger buses who are within the informal economy in México seem to be especially vulnerable to various psychosocial consequences, including Burnout syndrome, however, the psychometric evidence for these psychosocial measures is practically nonexistent in this sector. For this reason, this study analyzes the internal consistency and validity of scores from the MBI-GS (Maslach Burnout Inventory, General Survey), in a sample of drivers of urban passenger buses in Mexico. Internal consistency was adequate for all three dimensions $(>.70)$. The Confirmatory Factor Analysis satisfactorily reproduced the trifactorial structure of the MBI-GS (CFI = .989, RMSEA $=.045$, SRMR $=.075$ ) and low to moderate correlations were observed with nonpsychotic psychiatric disorders (symptoms) (GHQ-28) thus confirming the criteria validity. The implications of the results are discussed.

Key words: Burnout, validity, confirmatory factor analysis, internal consistency, informal drivers of urban passenger transport.

\section{Introducción}

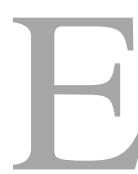

I trabajo como una actividad base en la producción de bienes y servicios es sin duda el eje del desarrollo en toda sociedad y su mercado se ofrece a voluntad de las personas mediante acuerdos, por lo que se puede ejercer formal o informalmente (STPS, 2007). La OIT (2014) asume como economía informal a las actividades de los trabajadores y unidades económicas que no cubren acuerdos formales dentro de un marco legal e incluyen dos rubros: el sector informal, caracterizado por las unidades económicas $o$ empresas privadas sin regulación alguna y ausencia de pago de impuestos; y el trabajo informal, que parte de las características del empleo de trabajadores que reciben un salario $\mathrm{y}$ mantienen relaciones de trabajo, pero no pueden hacer cumplir sus derechos laborales (Galindo, Mariana y Ríos, 2015; García, 2008; OIT, 2014). Entre la variedad de trabajos informales se pueden encontrar algunos prestadores de servicios domésticos, vendedores ambulantes, transportistas, etc., (García, 2008).

En particular, los estudios en la región latinoamericana han puesto en evidencia que los transportistas de pasajeros representan uno de los grupos de mayor riesgo a la salud ocupacional, debido a las consecuencias en la salud física y mental (e.g., enfermedades respiratorias, estrés, ansiedad, etc.) vinculados a varios tipos condiciones ambientales, por ejemplo exposición a factores derivados del proceso de trabajo (e.g., ruido, iluminación inadecuada, gases, polvos, etc.), exigencias ergonómicas (e.g., sobre-esfuerzo, posiciones incómodas, carga mental, etc.), o exposición a factores de riesgo psicosocial (Bonilla y Gafaro, 2017; Rosales, Mendoza y Granados, 2018; Tovalin y Lazcano, 1991).

A diferencia de lo que ocurre en países desarrollados, en Latinoamérica existen básicamente dos tipos de 
transportistas o conductores de pasajeros, unos ejercen formalmente dentro de empresas privadas de transporte foráneo, cuyas trayectorias pueden recorrer entidades federativas o estatales y están constituidos por autobuses modernos; o por otro lado, aquellos del transporte urbano de pasajeros que ejercen informalmente por concesiones otorgadas por el gobierno, y generalmente se constituyen por taxis y camiones no tan modernos, cuya trayectoria se limita a municipios o ciudades (INEGI, 2013; Lóyazana y Curiel, 2015). Algunos estudios han demostrado que los segundos son particularmente más sensibles a diversas condiciones psicosociales y de estrés (Lima y Juárez, 2008).

Una de las posibles consecuencias negativas a la salud en los conductores es el síndrome de burnout (SB). Recientemente en una entrevista (Juárez, 2014), la pionera en investigación sobre el SB Christina Maslach, lo define como "un conjunto de experiencias interrelacionadas en las que incluyen el agotamiento como respuesta al estrés, un cambio negativo de trato con indiferencia hacia el trabajo y con los demás, de ahí, impacta en las motivaciones para trabajar y generan percepciones negativas de la persona misma; a su vez afecta el desempeño laboral, la calidad en la que hacen su trabajo, sus asistencias al trabajo, problemas en la salud, etc." , concepto que se sostiene en revisiones de los avances del tema en décadas (Schaufeli, Leiter, \& Maslach, 2009).

Para la evaluación del SB, una de las herramientas más frecuentemente utilizadas en contextos latinoamericanos es el Maslach Burnout Inventory (MBI) (Juárez, Idrovo, Camacho y Placencia 2014; Maslach, Jackson \& Leiter, 1997). Existen tres (3) versiones oficiales: el MBI-HSS, Human Services Survey; el MBI-ES, Educators Survey; y el MBI-GS, General Survey (Maslach, Jackson, \& Leiter, 2010). Este último es más recomendable (Juárez, 2014; Schaufeli, et. Al. 2009) para la evaluación genérica en cualquier tipo de ocupación, y representa una mejor evolución conceptual y operacional del SB en comparación a otras versiones, empero ha sido utilizada escasamente.

En México, no hay de estudios del SB en conductores del transporte urbano de pasajeros, sin embargo, en otros países existen evidencias recientes predominantemente de Perú. Por ejemplo, en un estudio sobre la presencia de burnout en conductores peruanos (Salazar, y Pereda, 2010, $\mathrm{n}=237$ ) se reporta prevalencias altas de Agotamiento Emocional (31.2\%), de indiferencia (27.4\%), de baja Eficacia personal (47.3\%), y en conjunto, $13.5 \%$ presentó el SB. Así mismo, se observó una relación entre el agotamiento emocional con respecto a la "Situación de tráfico" y "Al conducir un automóvil". Análogamente en el estudio de Chunga-Trigozo et al., (2020) se reporta en una muestra de 50 conductores de taxi niveles altos de Agotamiento Emocional (70\%), de Indiferencia (66\%), de Eficacia Personal (44\%) y del SB (54\%); asimismo, una relación entre el estrés y el SB. En el estudio de Arias, Mendoza y Masías, (2013) se reportó en una muestra de 94 conductores, siendo que el $54.3 \%$ obtuvieron niveles altos del SB, de Agotamiento emocional (78.7\%), de 
Indiferencia (88.3\%) y de Eficacia personal $(37.2 \%)$.

No obstante, estos estudios como muchos otros en América Latina tienden a mantener instrumentos con lineamientos metodológicos, apropiaciones pragmáticas y praxis de EUA, Europa y otros países (Díaz y Gomes, 2001). En este sentido, es riesgoso hacer una inducción de la validez psicométrica de instrumentos desarrollados en poblaciones españolas o norteamericanas sin corroborarla en otra población o contexto (Díaz y Gomes, 2001; Merino-Soto; Angulo-Ramos, 2020), de tal manera que surge la necesidad de evidencia de la estructura psicométrica del MBI-GS en muestras y escenarios en contextos específicos, siguiendo las recomendaciones metodológicas que aluden a definir adecuadamente la versión, origen de adaptación y traducción; así como también reportar la consistencia interna en cada muestra y estimar la validez de constructo mediante el análisis factorial confirmatorio (AFC, Juárez, et. al, 2014). La implementación del AFC es altamente recomendado para saber si un modelo de medición hipotetizado de algún instrumento refleja en el comportamiento de varios parámetros de los ítems y sus variables latentes (Bentler \& Dougeon, 1996; Hair, Anderson, Tatham \& Black, 2006).

En Latinoamérica, solo se han encontrado 4 estudios dedicados a evidenciar la estructura latente del MBI-GS mediante la metodología de ecuaciones estructurales, específicamente usando el Análisis Factorial Confirmatorio (AFC). Entre ellos, el estudio de Juárez, Merino,
Fernández, Flores, Caraballo y Camacho (2020), cuyos resultados confirman validez transcultural y funcionamiento diferencial entre países en muestras de docentes. De igual manera, existen estudios (Fernández, Juárez y Merino, 2015a; Fernández, Merino y Guimet, 2015b; Flores, Merino, Camacho, Juárez y Placencia, 2015; Guevara y Ocampo, 2014) donde se replicó satisfactoriamente el modelo de tres dimensiones. Empero, algunos requirieron correlaciones de errores en algunos ítems y la remoción del ítem 13; a su vez, la consistencia interna mostró niveles aceptables ( $\alpha .>0.70)$. Analógicamente, el estudio de Pando, Aranda y López (2015) incorporó ocho países Latinoamericanos (incluyendo trabajadores inespecíficos de nacionalidad mexicana), los resultados no coinciden con la estructura tridimensional obteniendo dos de tres factores y una pobre consistencia interna en la mayoría de los países $(\alpha>.60)$. En este último, como el resto de la literatura (Guevara y Ocampo, 2016; Millan y D'Aubeterre, 2012), han realizado técnicas de validez fuera de las recomendaciones metodológicas (Díaz y Gomes, 2001; Juárez, et. al, 2014) utilizando solo análisis factorial exploratorio con métodos no recomendados $\mathrm{y} / \mathrm{o}$ instrumentos traducidos para otros contextos a la muestra.

Particularmente los estudios en revistas arbitradas referente a la validez de la versión del MBI-GS en conductores del transporte urbano no fueron encontrados. Por ende, se pone en duda los resultados de las exploraciones previas de prevalencia del SB en conductores ya que no cuentan con el respaldo de la validez del instrumento en dicha población, enfatizando la 
problemática en la recopilación de la información, como menciona Corral (2009, p. 229) "Siempre que se quiere recopilar información a fin de ayudarnos a tomar decisiones, nos enfrentamos al problema de qué tipo de instrumento usar o si realmente hay un cuestionario que nos ayude a tomar la decisión".

Por todo lo anterior, el presente estudio tiene tres objetivos, el primero, determinar la validez de constructo del MBI-GS mediante un Análisis Factorial Confirmatorio, el segundo, establecer la consistencia interna mediante los coeficientes Omega HA y Alfa de Cronbach, y el tercero, determinar la validez de criterio analizando las correlaciones entre los puntajes de las dimensiones del MBI-GS y del GHQ-28 como indicador de salud mental (Goldberg y Hillier, 1979) en conductores informales del transporte urbano de pasajeros. De esta manera, la importancia del estudio radica en su contribución al campo de la medición en la Psicología de la Salud Ocupacional

\section{Método}

Diseño. Se trata de un estudio observacional, transversal e instrumental (Montero y León, 2005).

Participantes. La muestra se obtuvo de manera no probabilística y por conveniencia, conformada por 201 conductores informales del transporte urbano de pasajeros del Estado de Morelos en México, el 100\% de sexo masculino con edades de entre 19 y 63 años (de 19 a 30 años $=28 \%$; $>30$ hasta 40 años $=41 \%$; $>40$ hasta 66 años=31\%) que llevan trabajando en la labor de conducción de entre 4 meses hasta 42 años (de 4 meses hasta 8 años=26\%; >8 hasta 15 años=32\%;>15 hasta 20 años=20\%; >20 hasta 42 años $=22 \%$ ). Los mismos fueron invitados a participar voluntariamente e informados sobre el anonimato y la confidencialidad de sus respuestas, autorizando con su firma el consentimiento informado para la participación.

Los criterios de inclusión fueron los siguientes: ser conductor del transporte urbano de pasajeros (no ayudante o asistente), participación libre y voluntaria confirmada con su firma en el consentimiento informado y que haya respondido la totalidad de respuestas en el cuestionario. No hubo criterios de exclusión, y vale la pena señalar que el GHQ28 no se consideró como criterio de exclusión, sino como criterio de validación del MBI-GS, aunque tampoco se presentó ningún caso con sintomatología clínica severa.

Instrumentos. Maslach Burnout Inventory - Encuesta General. (MBI-GSC) con autorización de la editorial Mind Garden) (Maslach, et al., 2010). Se utilizó la versión adaptada y traducida al español para América Latina por Juárez, et al., (2020). Está conformado por 16 ítems organizados en 3 dimensiones: Agotamiento Emocional (AGO, cinco ítems), Indiferencia (IN, cinco ítems) y Eficacia Personal (EP, seis ítems). Los ítems tienen una valoración ordinal de siete puntos de frecuencia, desde 0 (nunca) hasta 6 (todos los días). 
General Health Questionnaire - 28 (GHQ-28; Goldberg \& Hillier, 1979). Cuestionario que miden síntomas o trastornos psiquiátricos (no psicóticos). La versión aquí utilizada fue validada en México por Medina, Padilla, Campillo, Mas, Ezbán, Caraveo y Corona, (1983) presentando una varianza acumulada del $52.4 \%$, en las cuatro subescalas de 7 siete ítems cada una: Síntomas Somáticos (A); Ansiedad e Insomnio (B); Difusión Social (C) y Depresión Severa (D). Los ítems tienen una valoración ordinal de cuatro puntos de frecuencia que varían según el ítem, pueden ir desde "Más que antes" hasta "Mucho menos que antes"; desde "No" hasta "Mucho más que antes"; desde "Menos que antes" hasta "Mucho más que antes"; o desde "Mejor que antes", hasta "Mucho peor que antes". En el presente estudio, las escalas Síntomas Somáticos (A, $\alpha=.864$, IC95\% = $.803, .873 ; \omega=.863$, IC95\% $=.809, .895)$, Ansiedad e Insomnio (B, $\alpha=.870$, IC95\% $=$ $.832, .892 ; \omega=.872$, IC95\% = .829, .902), Difusión Social (C, $\alpha=.819$, IC95\% = .772, $.852 ; \omega=.818$, IC95\% $=.734, .867) \mathrm{y}$ Depresión Severa (D, $\alpha=.854$, IC95\% = $.816, .881 ; \omega=.852$, IC95\% $=.698, .913$ ) presentaron valores mayores a .70 en los coeficientes $\alpha$ y $\omega$.

El GHQ-28 es el único instrumento disponible para valorar sintomatología de trastornos psiquiátricos que ha sido validado en México, el cual utilizó en su validación entrevistas psiquiátricas como criterio, además de su Análisis Factorial (Medina, et. al, 1983). La elección de este criterio se debe primordialmente que el SB resulta ser un síndrome producto del estrés crónico que puede a su vez traer como consecuencias la presencia de alteraciones en la salud mental como la sintomatología somática, trastornos del sueño, ansiedad, depresión y dificultades en las relaciones sociales (Apiquian, 2007; FigueiredoFerraz, et. al, 2013; Juárez, 2014; Linares y Gutiérrez, 2010; Rocha, 2002; Uribe Prado, et. al, 2014).

Procedimiento. Se aplicaron los cuestionarios a lápiz y papel por estudiantes entrenados que no tenían ninguna relación previa con la organización de transporte donde se efectuó el estudio. La aplicación se realizó dentro de las terminales estableciendo grupos de conductores conforme sus horarios lo permitieron y en espacios disponibles que no causaran intensa intrusión en sus actividades, esto siempre y cuando el participante haya aceptado el consentimiento informado y entendido el anonimato. No hubo tiempo límite de respuesta, sin embargo, el tiempo máximo en demorarse a responder fue aproximadamente de una hora.

Análisis de datos. Se estimaron estadísticos descriptivos de los ítems utilizando el programa SPSS 25 . Se utilizó la distancia $D^{2}$ (Mahalanobis, 1936) para la identificación de valores extremos, además, se utilizó el programa normtest (DeCarlo, 1997) para establecer la normalidad multivariante de los datos.

Con el propósito de encontrar la evidencia de la validez de constructo del MBI-GS se aplicó la metodología de ecuaciones estructurales (SEM) mediante el Análisis Factorial Confirmatorio (Jöreskog, 1969) utilizando el programa EQS 6.3 
(Bentler \& Wu, 2012). Para representar la continuidad de latente de los ítems se utilizaron correlaciones policóricas (Holgado, Chacón, Barbero \& Villa, 2010; Morata-Ramirez \& Holgado-Tello, 2013). Del mismo modo, se aplicó el método máxima verosimilitud robusto, que ajusta el error estándar de los parámetros y la prueba $\chi^{2}$ para disminuir el impacto de la no normalidad de los ítems $\left(S B-\chi^{2}\right.$; Satorra \& Betler, 1994).

Debido a que el ítem 13 ha evidenciado un peso factorial bajo respecto a su constructo en las traducciones españolas e investigaciones internacionales (incluyendo aquellas en contextos latinos), su exclusión es la recomendación más común (Bakker, Demerouti, \& Schaufeli, 2002; Chirkowska-Smolak \& Kleka, 2011; Fernández, et. al, 2015a; Fernández, et., al, 2015b; Gil-Monte, 2002; Juárez, Merino, et al., 2020; Oramas, González, y Vergara, 2007; Salanova \& Schaufeli, 2000; Schutte, Toppinnen, Kalimo \& Schaufeli, 2000). Sin embargo, el presente estudio considera importante el reporte del análisis en presencia y ausencia del ítem, visto que existe una nula evidencia psicométrica en esta población en particular.

En el procedimiento del AFC se incorporaron especificaciones estructurales habituales: a) covariación cero entre los términos de error de los ítems y los factores, y b) cada ítem fue influenciado por una única variable latente. Para detectar posibles modificaciones del modelo de medición evaluado, se implementaron los índices de Lagrange (IM; Sörbom, 1989). La interpretación de los potenciale IM se basó en la significancia estadística, y significancia lógica y coherencia conceptual correspondiente (Boomsma, 2000). En cuanto la significancia práctica del ajuste fue evaluada mediante distintos indicadores como el Comparative Fit Index, CFI > 0.95 (Bentler, 1990), el Root Mean Square Error of Approximation, RMSEA $\leq 0.05$ (Steiger \& Lind, 1980), y el Standardized Root Mean Square Residual, SRMR $\leq 0.08$ (Jöreskog \& Sörbom, 1986).

Por otro lado, se estimó la consistencia interna mediante el coeficiente $\omega$ (McDonald, 1999). El modelamiento de este último es adecuado para la fiabilidad, siendo los cálculos más estables debido a que trabaja con las cargas factoriales y no depende del número de ítems (Revelle \& Zinbarg, 2008; Ventura y Caycho, 2017). El $\omega$ fue calculado mediante la herramienta "HA" macro Omega (Hayes \& Coutts, 2020) fundamentada por la metodología de la solución close-form (Hancock \& An, 2020), cuyo cálculo se basa en las covarianzas de las respuestas de los ítems y la varianza de la suma de los ítems, método comprobado en comparación con las cargas del AFC y brinda resultados equiparables, por lo que resulta una alternativa para estimar la confiabilidad $\omega$ bajo una dimensionalidad. Para propósitos de comparación y que es habitual su cálculo en las investigaciones psicométricas, se estimó el coeficiente $\alpha$ (Cronbach, 1951).

En el caso de la validez de criterio, se utilizó el coeficiente correlacional rho de Spearman adecuado para muestras no normales y de tipo ordinal (Hernández, Fernández y Baptista, 2014). Se correlacionó la puntuación de cada una de 
las escalas (síntomas somáticos, ansiedad e insomnio, difusión social y depresión grave) del GHQ-28 (Cuestionario de Salud General) con los puntajes de las dimensiones correspondientes al MBI-GS.

\section{Resultados}

Los análisis preliminares consistieron en una exploración de los datos. En relación con el valor crítico para la identificación de valores extremos mediante la distancia de Mahalanobis $\left(D^{2}=\right.$ 568.5058), se identificaron 10 casos que superan el valor crítico (Bonferroni- $\alpha=$ $.05 / n=40.43$ ), el caso más pequeño de los identificados tiene un valor de 41.04, mientras que el mayor tiene un valor de 55.69. De esta manera se decidió retirarlos de los análisis, quedando con una muestra 191 conductores. En cuanto a las pruebas de normalidad (DeCarlo, 1997) dieron como resultado una discrepancia con la hipótesis de normalidad, este supuesto se espera para la aplicación de ecuaciones de modelos estructurales, por lo que los estadísticos implementados atenúan esta condición y evitan sesgos en los estadísticos de ajuste.

\section{Análisis Factorial Confirmatorio}

Se probó la estructura trifactorial obteniendo diversos índices de ajuste (Tabla 1) y se estimaron cargas factoriales $\mathrm{y}$ correlaciones interfactoriales con sus respectivos intervalos de confianza (Tabla 2). Se valoraron 3 modelos distintos debido a que el modelo original (Modelo 1, Tabla 1), no presentó inicialmente un ajuste estadístico satisfactorio, siendo además que el ítem 13 no ofrece una contribución importante al modelo de acuerdo con su carga factorial y varianza explicada en su factor IN. Por tanto, se estableció el Modelo 2 en el que se retiró el ítem 13, cuyos índices de ajuste mostraron un mejoramiento moderado en el modelo, no obstante, siguieron presentándose discrepancias en el índice RMSEA, además que el SRMR apenas alcanza el valor recomendado.

Tabla 1

Evaluación de los modelos para el MBI-GS

\begin{tabular}{ccccccc}
\hline Modelo factorial & $\boldsymbol{S B}-\boldsymbol{\chi}^{2}$ & CFI & RMSEA & \multicolumn{2}{c}{ IC (90\%) } & \multirow{2}{*}{ SRMR } \\
\cline { 5 - 6 } & & & & LI & LS & \\
\hline $\begin{array}{c}\text { Modelo 1 } \\
\text { (gl: 120) }\end{array}$ & $3068.099^{* *}$ & .955 & .083 & .069 & .097 & .104 \\
$\begin{array}{c}\text { Modelo 2 } \\
\quad \text { (gl: 84) }\end{array}$ & $138.9358^{* *}$ & .981 & .059 & .041 & .075 & .079 \\
$\begin{array}{c}\text { Modelo 3 } \\
\text { (gl: 83) }\end{array}$ & $115.1258^{* *}$ & .989 & .045 & .022 & .064 & .075 \\
\hline
\end{tabular}

Notas: Modelo 1 = todos los ítems; Modelo 2 = sin el ítem 13; Modelo 3 = sin el ítem13 y una correlación entre los errores de los ítems 14 y 15; $S B-\chi^{2}=$ Satorra \& Betler Chi-Square; gl = grados libertad; CFI = Comparative Fit Index; RMSEA = Root Mean Squared Error Aproximation; LI = límite inferior; LS = límite superior; SRMR = Standardized Root Mean Square Residual; ${ }^{* *} p<.01$. 
De este modo, examinando los índices de modificación se encontró una reespecificación que produce un sustancial cambio en el SB- $\chi 2$ (IM $=63.525, p_{\text {Hancock }}<$. 001) con respecto al modelo 2 , consiste en una correlación de los errores de los ítems 14 ("Dudo de la relevancia que tiene $\mathrm{mi}$ trabajo") y 15 ("Me he vuelto más indiferente con respecto a si mi trabajo contribuye en algo"), estableciendo así el modelo 3. Dichos ítems reflejan de manera conceptual una actitud de indiferencia hacia el propio trabajo, así como a su valor y significado (Fernández, et. al, 2015; Maslach et al., 1997), por lo que la correlación tiene viabilidad teórica. En la modificación realizada se obtuvo una

Tabla 2

Cargas factoriales del AFC del MBI-GS en 3 modelos correlación de magnitud moderada entre los errores de estos ítems $(r=.578, \operatorname{cov}=$ $3.69, p<.01$ ), la cual produjo una mejora sustancial en los índices de ajuste (Tabla 1). Respecto a las cargas factoriales del Modelo 2 y 3, la mayoría indican que los ítems adecuan congruentemente con los factores; cabe mencionar que el ítem cinco se encuentra ligeramente por debajo de .40, sin embargo, se reconoce su importancia teórica en el modelo. De igual forma, en el modelo 3 (Tabla 2) se manifestaron correlaciones interfactoriales altas entre AGO e IN $(r=.897, p<.01)$, IN y EP ( $r=-$ $.786, p<.01)$ y una moderada entre AGO y $\mathrm{EP}(r=-.229, p<.01)$.

\begin{tabular}{|c|c|c|c|c|c|c|c|c|c|c|c|c|}
\hline \multirow[t]{2}{*}{ Ítems } & \multicolumn{4}{|c|}{ Modelo 1} & \multicolumn{4}{|c|}{ Modelo 2} & \multicolumn{4}{|c|}{ Modelo 3} \\
\hline & AGO & EP & IN & $h^{2}$ & AGO & EP & IN & h2 & AGO & EP & IN & $h^{2}$ \\
\hline $\mathrm{V} 1$ & .707 & & & .500 & .707 & & & .500 & .708 & & & .501 \\
\hline V2 & .653 & & & .427 & .654 & & & .427 & .657 & & & .431 \\
\hline V3 & .761 & & & .580 & .762 & & & 580 & .762 & & & .581 \\
\hline V4 & .784 & & & .615 & .784 & & & .615 & .785 & & & .617 \\
\hline V6 & .802 & & & .643 & .801 & & & .642 & .799 & & & .638 \\
\hline V5 & & .389 & & .151 & & .389 & & .151 & & .388 & & .150 \\
\hline V7 & & .528 & & .279 & & .529 & & .280 & & .527 & & .277 \\
\hline V10 & & .760 & & .578 & & .760 & & .578 & & .760 & & .577 \\
\hline V11 & & .880 & & .775 & & .880 & & .775 & & .882 & & .778 \\
\hline V12 & & .744 & & .554 & & .744 & & .553 & & .744 & & .554 \\
\hline V16 & & .736 & & .541 & & .736 & & .541 & & .735 & & .540 \\
\hline V8 & & & .906 & .820 & & & .906 & .821 & & & .904 & .818 \\
\hline V9 & & & .940 & .884 & & & .939 & .881 & & & .951 & .904 \\
\hline V13 & & & $\begin{array}{c}- \\
.206\end{array}$ & .043 & & & - & - & & & - & - \\
\hline V14 & & & .536 & .287 & & & .540 & .291 & & & .500 & .250 \\
\hline V15 & & & .695 & .483 & & & 698 & .487 & & & .675 & .456 \\
\hline
\end{tabular}




\begin{tabular}{|c|c|c|c|c|c|c|c|c|c|}
\hline \multicolumn{10}{|c|}{ Correlación interfactorial } \\
\hline & & \multicolumn{2}{|l|}{$\begin{array}{c}\text { IC } \\
95 \%\end{array}$} & & \multicolumn{2}{|c|}{ IC 95\% } & \\
\hline & & LI. & LS & & LI & LS & & LI & LS. \\
\hline EP- & $0<?$ & -.010 & - & - & -.067 & - & - & - & - \\
\hline AGO & -.002 & & .114 & .227 & & .387 & .229 & .065 & .393 \\
\hline IN- & .447 & .359 & .535 & & .892 & .892 & .897 & .897 & .897 \\
\hline AGO & .447 & & & 892 & & & & & \\
\hline IN-EP & -.198 & -.109 & - & - & -.762 & - & - & - & - \\
\hline
\end{tabular}

Notas: Modelo 1 = todos los ítems; Modelo 2 = sin el ítem 13; Modelo 3 = sin el ítem13 y una correlación entre los errores de los ítems 14 y 15; AGO = agotamiento emocional; $\mathrm{EP}$ = eficacia personal; IN = indiferencia; $\mathrm{LI}$ = límite inferior; LS = límite superior.

\section{Consistencia interna}

Los resultados obtenidos para la consistencia interna entre el coeficiente $\alpha$ y $\omega$ (Tabla 3) arrojaron valores similares entre sí en el caso de AGO, en EP. En el caso de IN se enfatiza una diferencia importante en el coeficiente $\alpha$ al retirar el ítem 13 (IN s/v13) obteniendo supremacía en valor de este último. Cabe mencionar que al utilizar la herramienta "HA" macro Omega se implementa una metodología (Hancock \& An, 2020) en la que es necesario que no se presenten debilidades de varianza y/o las correlaciones nulas con respecto a un ítem y el resto de una dimensión, lo cual detonaría imposible calcular el coeficiente hasta la ausencia del ítem inoperante, tal es el caso del IN con el ítem 13, en cambio, en IN(s/v13) se obtuvieron los valores mostrando supremacía en el $\alpha$ comparativamente al $\omega$.

Tabla3

Consistencia interna mediante los coeficientes alfa y el omega

\begin{tabular}{cccccccc}
\hline & \multirow{2}{*}{$\alpha$} & \multicolumn{2}{c}{ IC 95\% } & & \multicolumn{2}{c}{ IC 95\% } \\
\cline { 3 - 4 } & & LI & LS & & LI & LS \\
\hline AGO & .871 & .839 & .898 & .872 & .837 & .900 \\
EP & .740 & .679 & .793 & .737 & .654 & .794 \\
IN & .720 & .652 & .778 & & - & - & - \\
IN(s/v13) & .821 & .776 & .859 & & .799 & .711 & .858 \\
\hline
\end{tabular}

Notas: $\alpha=$ coeficiente alfa de Cronbach; $\omega=$ Coeficiente Omega; AGO = agotamiento emocional; EP = eficacia personal; IN = indiferencia; IN(s/v13) = Indiferencia sin el ítem 13; LI = límite inferior; LS = límite superior.

\section{Validez de Criterio}

Para corroborar la validez de criterio del MBI-GS se estimó el coeficiente rho de Spearman entre las puntuaciones de las escalas de este último con las del GHQ-
28, aunque en el caso de la subescala IN, se probó con y sin el ítem 13 (Is/v13) para enriquecer la evidencia de los resultados obtenidos en AFC del presente estudio. En cuanto a las correlaciones entre las subescalas del MBI-GS y el GHQ-28 fueron 
estadísticamente significativas. Como se observa en la Tabla 4, para el caso de AGO, se encontraron correlaciones altas con respecto a Síntomas Somáticos y Ansiedad e Insomnio, y moderadas con Difusión Social y Depresión Severa; estas fueron positivas, lo que indica que, a mayor AGO mayor será la presencia de indicadores de trastornos psiquiátricos. En el caso de EP, se encontraron correlaciones moderadas entre Difusión Social y Depresión Severa, y bajas con respecto a Síntomas Somáticos y Ansiedad e Insomnio, estas fueron negativas, lo que indica que a mayor

presencia de EP menor presencia de trastornos psiquiátricos. Respecto a la subescala completa de IN se encontraron correlaciones positivas moderadas entre todas las subescalas del GHQ-28, lo que indican que a mayor presencia de IN menores indicios de trastornos psiquiátricos. Comparativamente, las correlaciones de la subescala IN y las subescalas del GHQ-28 manifestaron cambios importantes sin la presencia del ítem 13 (INs/v13), obteniendo valores de correlación notoriamente mejores (Tabla $4)$.

Tabla 4

Correlaciones entre las subescalas del MBI-GS Y GHQ-28 (Validez de Criterio)

\begin{tabular}{lllll}
\hline & $\begin{array}{l}\text { Síntomas } \\
\text { Somáticos }\end{array}$ & $\begin{array}{l}\text { Ansiedad e } \\
\text { Insomnio }\end{array}$ & $\begin{array}{l}\text { Difusión } \\
\text { Social }\end{array}$ & $\begin{array}{l}\text { Depresión } \\
\text { Severa }\end{array}$ \\
\hline AGO & $.556^{* *}$ & $.570^{* *}$ & $.232^{* *}$ & $.224^{* *}$ \\
EP & $-.170^{*}$ & $-.182^{*}$ & $-.354^{* *}$ & $-.202^{* *}$ \\
IN & $.383^{* *}$ & $.375^{* *}$ & $.240^{* *}$ & $.339^{* *}$ \\
IN(s/v13) & $.439^{* *}$ & $.433^{* *}$ & $.304^{* *}$ & $.371^{* *}$ \\
\hline
\end{tabular}

Notas: $p>0.05^{*} ; p<0.01^{* *} ; \mathrm{AGO}=$ agotamiento emocional; $\mathrm{IN}=$ indiferencia; $\mathrm{EP}=$ eficacia personal; IN(s/v13) = Indiferencia sin el ítem 13 .

\section{Discusión}

El objetivo de este estudio fue evidenciar la validez de constructo de los puntajes del MBI-GS en una muestra de conductores de transporte urbano mediante la validez factorial y la consistencia interna, además de determinar la validez de criterio tomando como referencia los síntomas o trastornos psiquiátricos (no psicóticos) operacionalizado mediante el GHQ-28. En cuanto a la validez de constructo se refiere, en un primer análisis los resultados del AFC arrojan que el modelo original de 16 ítems del MBI-GS no cumple con los parámetros necesarios para concluir su efectiva funcionalidad debido a problemas tanto en las cargas factoriales como en los índices de ajuste. Debido a esto se probó un segundo modelo sin el ítem 13 perteneciente a IN cuya carga factorial había sido prácticamente nula y otro tercer modelo que requirió de una re-especificación de correlación en los errores de dos ítems 
pertenecientes a IN, cuyos resultados muestran indicadores satisfactorios sobre la validez de constructo en los puntajes observados del MBI-GS. Estos hallazgos de la estructura tri-factorial coinciden con otros estudios en muestras latinoamericanas que utilizaron la misma versión del MBI-GS (Fernández, et. al, 2015a; Fernández, et., al, 2015b; Flores, et. al, 2015; Guevara y Ocampo, 2014; Juárez, et. al, 2020).

De la misma forma, en las correlaciones interfactoriales en el modelo 3 en este estudio se manifestaron fuertes vinculaciones entre AGO e IN (.897) siendo las más alta, seguida de IN y EP (-.786) y moderadamente entre AGO y EP (-.229). Comparativamente, la presencia de estos resultados es uniforme a la evidencia latinoamericana en docentes de 3 países distintos donde implementó la validez transcultural del instrumento utilizado (Juárez et al., 2020).

Respecto a la covariación entre los errores de los ítems 14 ("Dudo de la relevancia que tiene mi trabajo") y 15 ("Me he vuelto más indiferente con respecto a si mi trabajo contribuye en algo"), puede deberse a una relación adicional a la varianza común de su constructo (IN) ya que ambos reflejan de manera conceptual una actitud de indiferencia hacia el valor y significado del propio trabajo, este requerimiento no resulta un problema mayor ya que se encuentra en el mismo factor (Byrne, 2012). Otra posible explicación que requiere futura investigación, es la posibilidad de una subdimensión de IN, ya que existen 2 estudios en el que se realizó la misma re- especificación en los mismos ítems (Fernández, et. al, 2015a; Fernández, et., al, 2015b;), pero son necesarios más estudios al respecto.

Referente a las estimaciones de consistencia interna se obtuvieron en todos los factores puntuaciones satisfactorias (> .70). Los resultados obtenidos de los coeficientes de consistencia interna $\alpha$ y $\omega$ presentaron valores similares en el caso AGO y EP. Cabe mencionar que la implementación de la herramienta "HA" macro Omega conlleva requerimientos robustos para su cálculo debido a que la metodología se basa en las covarianzas consistentes de las respuestas de los ítems y la varianza homogénea de la suma de los ítems (Hancock \& An, 2020), lo cual detona imposible su cálculo hasta la ausencia del ítem inoperante, tal es situación del ítem 13 en IN. En este caso (IN s/v13), se obtuvo supremacía en valor de $\alpha$ comparativamente con el $\omega$. En general, se encontraron elementos suficientes para considerar una óptima consistencia interna del MBI-GS.

En lo que a la validez de criterio se refiere, se encontraron asociaciones significativas entre las escalas del GHQ y las dimensiones que conforman el SB. Las correlaciones más fuertes en AGO respecto a Síntomas Somáticos $(r=.556)$ y Ansiedad e Insomnio ( $r=.570)$, las segundas más fuertes corresponden IN(s/v13) Síntomas Somáticos $(r=.439)$ y Ansiedad e Insomnio $(r=.433)$. En el caso de EP, se encontraron correlaciones que oscilan ser moderadas entre Difusión social ( $r=-$.354) y Depresión severa $(r=-.202)$, y más bajas con Somáticos ( $r=-.170)$ y Ansiedad e Insomnio 
( $r=-.182)$. Los resultados son esperados, ya que el SB se entiende como respuesta al estrés (distrés) crónico (Maslach \& Jackson, 1981) y sus mecanismos vinculados al estrés terminan por impactar la salud mental (Juárez y Camacho, 2011).

Aunque es un tema polémico (Schaufeli \& Salanova, 2007), es importante notar el sentido positivo que tiene la EP en la escala del MBI-GS, la que al presentar niveles bajos se asume como una característica negativa que conforma el burnout. Tal y como se presenta, se concibe como un indicador de la relevancia, eficiencia y capacidad para realizar el trabajo, así como para la resolución de conflictos laborales por el trabajador, de manera que su relación no se esperaría que fuera muy fuerte con indicadores sintomatológicos de enfermedad mental, sino más bien con aquellos del bienestar en la relación con el trabajo (Maslach \& Jackson, 1981). En su aspecto teórico, esto explicaría las correlaciones bajas, sin embargo, son necesarios más estudios para valorar el sentido teórico de la subescala EP en el MBI-GS.

En general los resultados son comprables con estudios previos que estimaron la relación entre las dimensiones del SB y las del GHQ (García, Saéz y Llor, 2000; Ríos, Peñalver y Godoy, 2008), lo que concluye que esta anáfora de las correlaciones muestra solidez en la validez de la estructura psicométrica. Una observación importante a tratar es la relevancia del ítem 13, ya que en todos los resultados de los análisis denotó precariedad psicométricamente hablando, de manera análoga se presenta en los distintos estudios psicométricos encontrados tanto en las traducciones españolas y estudios internacionales (incluyendo estudios latinos) donde su peso factorial es bajo respecto a su constructo, complicación que las investigaciones han resuelto con su exclusión (Bakker, Demerouti, \& Schaufeli, 2002; Chirkowska-Smolak \& Kleka, 2011; Fernández, et., al, 2015a; Fernández, et., al, 2015b; Gil-Monte, 2002; Juárez, Merino, et al., 2020; Oramas, González, y Vergara, 2007; Salanova \& Schaufeli, 2000; Schutte, Toppinnen, Kalimo \& Schaufeli, 2000). Existe alguna evidencia de que ítem 13 ("Sólo quiero hacer mi trabajo sin ser molestado") no presenta anomalías ni diferencias de traducción en 3 países latinoamericanos distintos (Juárez, et. al, 2020), lo que demuestra que su insuficiencia psicométrica puede deberse a otras situaciones que no permiten medir lo que se pretende en la versión original. Por tanto, a la futura presencia del ítem 13 se le debe considerar una reformulación del contenido o removerlo definitivamente, pues tanto la evidencia existente como la presente continúan cuestionando su funcionalidad.

De esta forma, los resultados obtenidos en el presente estudio en la validez de criterio, la validez de constructo y la consistencia interna, dan méritos a la conclusión general de un adecuado funcionamiento psicométrico de los puntajes del MBI-GS en la muestra de conductores de transporte urbano en este estudio, sin embargo, se recomienda valorar cuidadosamente la inclusión del ítem 13, por los problemas presentados. 
Las limitaciones del presente estudio sugieren los cuidados tradicionales en las interpretaciones de los resultados. En primer lugar, el tamaño muestral podría considerarse pequeño y afectar la potencia estadística, sin embargo, la muestra obtenida parece suficiente con relación a los ítems de la escala y los pesos factoriales obtenidos. En segundo lugar, no se incluyó una validez conceptual y semántica de los ítems en la muestra validando el contenido y sus significados. Visto que, la muestra está

\section{Referencias}

Álvarez-Cabrera, P., Pérez-Arqueros, W., Reyes-Soto, D., y Jofré-Portales, B. (2017). Síndrome de quemarse por el trabajo (SQT) y Estrés: funcionarios de locomoción colectiva de la ciudad de Arica, Chile. Revista Iberoamericana de Psicología, 10(2), 25-34. https://doi.org/10.33881/20271786.rip.10203

Apiquian A. (2017). El síndrome de burnout en las empresas. Presentación realizada en el Tercer Congreso de Escuelas de Psicología de las Universidades Red Anáhuac. Mérida, Yucatán.

Arias, W., Mendoza del Solar, L. y Masías, M. (2013). Síndrome de burnout en conductores de transporte público de la ciudad de Arequipa. Revista Peruana de Psicología y Trabajo Social, 2(2), 111-122. https://pdfs.semanticscholar.org/f 3a5/c12eea92fff44ad2d2404259ca ad758ed563.pdf? ga $=2.218233050$ conformada por conductores del transporte urbano de pasajeros pertenecientes al trabajo informal donde ha habido escases de evidencia psicométrica y metodologías limitadas, el presente estudio cubre esta necesidad en alguna medida. Sin embargo, quedan abiertas las puertas a futuros estudios la réplica cubriendo las limitantes presentes y su posible expansión a distintos tipos de ocupaciones en sectores informales no tradicionales.

\section{$.2119173035 .1594672816-$ $\underline{469300732.1575761873 .}$}

Bakker, A. B., Demerouti, E., \& Schaufeli, W. B. (2002). Validation of the Maslach Burnout Inventory - General Survey: An iInternet study. Anxiety, Stress, and Coping, 15(3), 245-260. https://www.wilmarschaufeli.nl/p ublications/Schaufeli/179.pdf.

Bentler, P. M. \& Dugeon, P. (1996). Covariance structure analysis: statistical practice, theory, and directions. Annual Review of Psychology, 47, 563-592. https://doi.org/10.1146/annurev. psych.47.1.563.

Bentler, P. M. (1990). Comparative fit indexes in structural models. Psychological Bulletin, 107(2), 238246. https://doi.org/10.1037/00332909.107.2.238.

Bentler, R. M. \& Wu, E. J. C. (2012). EQS for window (Version 6.2) [Statistical 
Program for Windows]. Multivariate Software, Inc.

Bonilla, L. y Gafaro, A. (2017). Condiciones laborales y riesgos psicosociales en conductores de transporte público. Revista Cubana de Salud y Trabajo, 18(2),48-56.

https://www.medigraphic.com/pd fs/revcubsaltra/cst2017/cst172h.pdf

Boomsma, A. (2000). Reporting analyses of covariance structures. Structural Equation Modeling, 7(3), 461-483. https://doi.org/10.1207/S153280 07SEM0703 6.

Byrne, B. M. (2012). Multivariate applications series. Structural equation modeling with Mplus: Basic concepts, applications, and programming. Routledge Taylor \& Francis Group.

Chirkowska-Smolak, T. \& Kleka, P. (2011). The Maslach Burnout InventoryGeneral Survey: Validation across different oOccupational groups in Poland. Polish Psychological Bulletin, 42(2), 86-94. https://doi.org/10.2478/v10059011-0014-X.

Chunga -Trigozo, L, Duarte -Gonzáles, E., Magallanes-Cabrera, J., TorresChávez, K., Verde-Gutiérrez, R., Rodríguez-Díaz, D. y MorilloArqueros, A. (2020). Relación entre estrés laboral y síndrome de burnout en conductores de taxi de la ciudad de Trujillo. Revista Médica Vallejiana, 9(1), 13-7. https://doi.org/10.18050/revista medicavallejiana.v9i1.2421.

Corral, Y. (2009). Validez yY confiabilidad de los instrumentos de investigación para la recolección de datos. Revista Ciencias De La Educación, 19(33), 228-247.

Cronbach, L. (1951). Coefficient alpha and the internal structure of test. Psychometrika, 16(3), 297-334.

De Carlo, L.T. (1997). On the meaning and use of kurtosis. Psychological Methods, 2(3), 292-307. https://doi.org/10.1037/1082989X.2.3.292

Díaz, F., y Gomes, I. (2001). La investigación del burnout en Latinoamérica entre 2000 y el 2010. Psicología desde el Caribe, 33(1), 113-131. http://dx.doi.org/10.14482/psdc.3 3.1.8065.

Fernández, M., Juárez, A., y Merino, C., (2015a). Análisis Estructural e invarianza de medición del MBI-GS en trabajadores peruanos. Liberabit, 21(1), 9-20. http://www.scielo.org.pe/pdf/libe r/v21n1/a02v21n1.pdf.

Fernández, M., Merino, C., y Guimet, M. (2015b). Propiedades psicométricas del Maslach Burnout Inventory General Survey en una muestra de docentes de Lima, Perú. Homenaje a Reynaldo Alarcón, 371391. Editorial Universal.

Figueiredo-Ferra H., Gil-Monte P., Ribeiro B., \& Grau-Alberola E. (2013) 
Influence of a Cognitive Behavioural Training Program on Health: A Study among Primary Teachers. Revista Psicodidáctica, 18(2), 343356.

Flores, C., Merino, C., Camacho, A., Juárez, A., \& Placencia, O. (2015). Síndrome de burnout en instructores comunitarios: propiedades psicométricas del Masclach Burnout Inventory General Survey (MBI-GS). Juárez-García A. (Eds.). Investigaciones psicométricas de escalas psicosociales en trabajadores mexicanos (pp. 279-308). Plaza y Valdés Editores.

García, B. (2008). La carencia de empleos satisfactorios: Una discusión sobre indicadores en B. El dato en cuestión: un análisis de las cifras socio-demográficas. El Colegio de México, 35-56.

García, M., Sáez, M., Llor, B., (2000). Burnout, satisfacción laboral y bienestar en personal sanitario de salud mental. Revista de Psicología del Trabajo y de las Organizaciones, 16(2), 215228.

Gil-Monte, P. (2002). Validez factorial de la adaptación al español del Maslach Burnout Inventory-General Survey. Salud pública de México, 44(1), 3340.

Goldberg, D. P. \& Hillier, V. F. (1979). A scaled version of the General Health Questionnaire. Psychological Medicine, $\quad 9, \quad 139-145$.
https://doi.org/10.1017/S003329 1700021644

Guevara, L. y Ocampo, N. (2014). Propiedades psicométricas de cConfiabilidad y vValidez del Maslach Burnout Inventory General Survey. Revista Interamericana de Psicología Organizacional, 33(2), 128-142. https://doi.org/10.21772/ripo.v33 $\underline{\mathrm{n} 2 \mathrm{a} 04}$

Hair, J. F., Anderson, R. E., Tatham, R. L., \& Black, W. C. (2006). Multivariate data analysis (6th ed.). Prentice Hall.

Hancock, G. R., \& An, J. (2020). A closed-form alternative for estimating omega reliability under unidimensionality. Measurement: Interdisciplinary Research and Perspectives, 18(1), 114.

https://doi.org/10.1080/1536636 7.2019.1656049

Hayes A. \& Coutts J. (2020). Use omega rather than Cronbach's alpha for estimating reliability. But..., Communication Methods and Measures, 1-24 https://doi.org/10.1080/1931245 $\underline{8.2020 .1718629}$

Hernández, R., Fernández, C. y Baptista, P. (2014). Metodología de la investigación ( $6^{a}$ ed). MCGraeHill.

Holgado, E.P, Chacón, S., Barbero, I. \& Vila, E. (2010). Polychoric versus Pearson correlations in exploratory and confirmatory analysis of ordinal variables. Quality and Quality, $\quad 44(1), \quad$ 153-166. 
https://doi.org/10.1007/s11135008-9190-y

INEGI, (2013). Encuesta anual de transportes: datos 2012: cifras definitivas / Instituto Nacional de Estadística y Geografía. INEGI, 53.

Jöreskog, K. G. \& Sörbom, D. (1986). LISREL VI: Analysis of linear structural relationships by maximum likelihood and least squares methods $\left(4^{a}\right.$ ed). Mooresville, IN: Scientific Software, Inc.

Jöreskog, K. G. (1969). A general approach to confirmatory maximum likelihood factor analysis. Psychometrika, 34(2), 183-202.

Juárez, A. (2014). Entrevista con Cristina Maslach: reflexiones sobre el síndrome de burnout. Liberabit, 20(2), 199-208. http://www.scielo.org.pe/scielo.ph p?script=sci arttext\&pid=S1729$\underline{48272014000200001}$

Juárez, A. y Camacho, A. (2011). Reflexiones teórico-conceptuales de los psicosocial en el trabajo. Universidad Autónoma del Estado de Morelos: Juan Pablos Editor.

Juárez-García, A., Idrovo, A., Camacho-Ávila y Placencia- Reyes, O. (2014). Síndrome de burnout en población mexicana: Una revisión sistemática. Salud Mental, 37(2),159-176.

Juárez-García, A., Merino-Soto, C., Fernández-Arata, M., FloresJiménez, C. A., Caraballo, M., y Camacho-Cristiá, C. (2020).
Validación transcultural y funcionamiento diferencial del mbigs en docentes de tres países latinoamericanos. Avances en Psicología Latinoamericana, 38(1), 135-156.

http://dx.doi.org/10.12804/revist as.urosario.edu.co/apl/a.6621

Lima, C. y Juárez, A. (2008). Un estudio exploratorio sobre estresores laborales en conductores de transporte público colectivo en el estado de Morelos, México. Ciencia y Trabajo, 10(30), 126-131.

Linares, L. y Gutierrez, R. (2010). Satisfacción Laboral y percepción de salud mental en profesores. Revista Mexicana de Investigación en Psicología, 2, 33-38.

Lóyazaga O. y Curiel, V. (2015). Los trabajadores del autotransporte. Alegatos, 91, 563- 590.

Mahalanobis, P.C. (1936). On the generalized distance in statistics. Proceedings of the National Institute of Science, Calcutta, 2(1), 49-55.

Maslach, C. \& Jackson, S. E. (1981). Maslach Burnout Inventory. Manual (2 ${ }^{\mathrm{a}}$ ed.). Palo Alto, CA: Consulting Psychologists Press.

Maslach, C., Jackson, S. E. \& Leiter, M. P. (1997). Maslach Burnout Inventory manual ( $3^{\text {a }}$ ed.). Palo Alto, California: Consulting Psychologists Press.

Maslach, C., Jackson, S. E., \& Leiter, M. P. (2010). Maslach Burnout Inventory: 
Manual and non-reproducible instrument and scoring guides ( 4 a ed.). California: Mind Garden.

McDonald, R. P. (1999). Test theory: A unified treatment. Lawrence Erlbaum Associates Publishes.

Medina-Mora, ME, Padilla, GP, CampilloSerrano, C., Mas, CC, Ezbán, M., Caraveo, J. y Corona, J. (1983). La estructura factorial del GHQ: una versión a escala para el servicio de medicina general de un hospital en México. Psychological Medicine, 13 (2), 355-361. https://doi.org/10.1017/S003329 $\underline{1700050984}$

Merino-Soto, C., \& Angulo-Ramos, M. (2020). Inducción de la validez: comentarios al estudio de validación del Compliance Questionnaire on Rheumatology. Revista Colombiana de Reumatología, (Carta al editor). https://doi.org/10.1016/j.rcreu.20 $\underline{20.05 .005}$.

Montero y León. (2005). Clasificación y descripción de las metodologías de investigación en Psicología. Revista Inter nacional de Psicología Clínica y de la Salud, 2(3), 503-508. http://www.redalyc.org/articulo.o a?id=33701007

Morata-Ramírez, M. \& Holgado-Tello, F. (2013). Construct validity of likert scales through confirmatory factor analysis: A simulation study comparing diferentedifferent methods of estimation based on
Pearson and polychoric correlations. International Journal of Social Science Studies, 1(1), 54-61. https://doi.org/10.11114/ijsss.v1i $\underline{1.27}$

OIT, Oficina Regional para América Latina y el Caribe. (2014). Panorama temático laboral: Transición a la formalidad en América Latina y el Caribe. Organización Internacional del Trabajo.

Olivares, V., Jélvez, C., Mena, L., y Lavarello, J. (2013). Estudios sobre burnout y carga mental en conductores del transporte público de Chile (Transantiago). Ciencia \& Trabajo, 15(48),

173-178. http://dx.doi.org/10.4067/S0718$\underline{24492013000300011}$

Oramas, A., González, A., y Vergara, A. (2007). El desgaste profesional. Evaluación y factorialización del MBI-GS. Revista Cubana de Salud y Trabajo, 8(1), 37-45.

Revelle W. \& Zinbarg R. (2008). Coefficients alpha, beta, omega and the glb: comments on Sijtsma. Psychometrika, 74(1), 145-154.

Ríos, M., Peñalver, F., y Godoy, C., (2008). Burnout $y$ salud percibida en profesionales de enfermería de cuidados Intensivos. Enfermería Intensiva, 19(4), 169-78.

Ríos, V. y Galindo, M. (2015). "Informalidad" en serie de estudios económicos, 1 , México ¿Cómo Vamos? http://www.mexicocomovamos.mx Lwp- 
content/uploads/2015/12/doc IN FORMALIDAD v1 FINAL.pdf

Rocha J. (2002). Sindrome de "Burnout" ¿El médico de urgencias incansable? Revista Mexicana de Medicina de Urgencias, 1(2), 48-56.

Rosales-Flores, R., Mendoza-Rodríguez, J. y Granados-Cosme, J. (2017). Condiciones de vida, salud y trabajo en conductores de taxi en la Ciudad de México. Revista Médica del Instituto Mexicano del Seguro Social, 56(3), 279-286. http://revistamedica.imss.gob.mx/ editorial/index.php/revista medica /article/view/1271

Salanova, M., \& Schaufeli, W. B. (2000). Exposure to information technologies and its relation to burnout. Behavior \& Information Technology, 19, 385-392. https://doi.org/10.1080/0144929 $\underline{00750000081}$

Salazar, S., y Pereda, E. (2010). Síndrome de burnout y patrones de comportamiento ante tráfico en conductores. Revista de Psicología, 12 , 141-169.

https://sisbib.unmsm.edu.pe/BVRe vistas/rev psicologia cv/v12 2010 /pdf/a07.pdf

Satorra, A., \& Bentler, P. M. (1994). Corrections to test statistics and standard errors in covariance structure analysis. In A. von Eye \& C. C. Clogg (Eds.), Latent variables analysis: Applications for developmental research (p. 399419). Sage Publications, Inc.

Schaufeli W., Leiter M., \& Maslach C. (2009). Burnout: 35 years of research and practice. Career Development International, 14(3), 204-220. https://doi.org/10.1108/1362043 0910966406

Schaufeli, W. B., \& Salanova, M. (2007). Efficacy or inefficacy, that's the question: Burnout and work engagement, and their relationships with efficacy beliefs. Anxiety, stress, and coping, 20(2), 177-196. https://doi.org/10.1080/1061580 $\underline{0701217878}$

Schutte, N., Toppinnen, S., Kalimo, R., \& Schaufeli, W. B. (2000). The factorial validity of the Maslach Burnout Inventory-General Survey across occupational groups and nations. Journal of Occupational and Organizational Psychology, 73, 5366.

Sörbom, D. (1989). Model modification. Psychometrika, 54, 371- 384. https://doi.org/10.1007/BF02294 $\underline{623}$

Steiger, J. H. \& Lind, J. M. (1984). Statistically based tests for the number of common factors. Annual meeting of the Psychometric Society.

STPS (2007). Seguridad y salud en el tTrabajo en México: Avances, retos y desafíos. Secretaria del Trabajo y Prevención Social. https://www.gob.mx/cms/uploads /attachment/file/279153/Libro- 
Seguridad y salud en el trabajo e $\underline{\mathrm{n} \text { Me xico- }}$

Avances retos y desafios Digital .pdf

Tovalin H. y Lazcano F. (1991). Las condiciones de salud de los conductores de autotransportes urbanos de pasajeros de la ciudad de México. Bol of Saint Panam, 111(4), 324-332. https://iris.paho.org/bitstream/ha ndle/10665.2/16588/v111n4p324 .pdf?sequence $=1$

Uribe, F., López P., Galicia, C. y García A. (2014). Síndrome de desgaste ocupacional (burnout) y su relación con salud y riesgo Psicosocial en funcionarios públicos que imparten justicia en México, D.F. Acta de investigación Psicológica, 4(2), 1510-1519.

Ventura-León J., Caycho-Rodríguez, T. (2017). El coeficiente omega: un método alternativo para la estimación de la confiabilidad. Revista Latinoamericana de Ciencias Sociales, Niñez y Juventud, 15(1), 625-627.

https://www.redalyc.org/pdf/773 /77349627039.pdf 